\title{
Microstructural Neuroimaging of Frailty in Cognitively Normal Older Adults
}

\author{
Qu Tian ${ }^{1 *}$, Owen A. Williams ${ }^{2,3}$, Bennett A. Landman ${ }^{4}$, Susan M. Resnick ${ }^{2}$ and \\ Luigi Ferrucci ${ }^{1}$
}

${ }^{1}$ Longitudinal Studies Section, National Institute on Aging, Baltimore, MD, United States, ${ }^{2}$ Laboratory of Behavioral Neuroscience, National Institute on Aging, Baltimore, MD, United States, ${ }^{3}$ Department of Experimental Psychology, University of Oxford, Oxford, United Kingdom, ${ }^{4}$ School of Engineering, Vanderbilt University, Nashville, TN, United States

OPEN ACCESS

Edited by:

Tzvi Dwolatzky

Technion Israel Institute of

Technology, Israe

Reviewed by:

Liang-Yu Chen,

Taipei Veterans General

Hospital, Taiwan

Scott Kehler

Dalhousie University, Canada

*Correspondence:

Qu Tian

qu.tian@nih.gov

Specialty section:

This article was submitted to

Geriatric Medicine,

a section of the journal

Frontiers in Medicine

Received: 28 March 2020 Accepted: 21 September 2020

Published: 23 October 2020

Citation:

Tian Q, Williams OA, Landman BA, Resnick SM and Ferrucci L (2020) Microstructural Neuroimaging of Frailty in Cognitively Normal Older

Adults. Front. Med. 7:546344

doi: 10.3389/fmed.2020.546344
Physical frailty is an age-related clinical syndrome that is associated with multiple adverse health outcomes, including cognitive impairment and dementia. Recent studies have shown that frailty is associated with specific volumetric neuroimaging characteristics. Whether brain microstructural characteristics, particularly gray matter, associated with frailty exist and what their spatial distribution is have not been explored. We identified 670 participants of the Baltimore Longitudinal Study of Aging who were aged 60 and older and cognitively normal and who had concurrent data on frailty and regional microstructural neuroimaging markers by diffusion tensor imaging (DTI), including mean diffusivity (MD) of gray matter and fractional anisotropy (FA) of white matter. We identified neuroimaging markers that were associated with frailty status (non-frail, pre-frail, frail) and further examined differences between three groups using multivariate linear regression (non-frail = reference). Models were adjusted for age, sex, race, years of education, body mass index, scanner type, and Apolipoprotein E e4 carrier status. Compared to the non-frail participants, those who were frail had higher MD in the medial frontal cortex, several subcortical regions (putamen, caudate, thalamus), anterior cingulate cortex, and a trend of lower FA in the body of the corpus callosum. Those who were pre-frail also had higher MD in the putamen and a trend of lower FA in the body of the corpus callosum. Our study demonstrates for the first time that the microstructure of both gray and white matter differs by frailty status in cognitively normal older adults. Brain areas were not widespread but mostly localized in frontal and subcortical motor areas and the body of the corpus callosum. Whether changes in brain microstructure precede future frailty development warrants further investigation.

Keywords: microstructure, neuroimaging, diffusion tensor imaging, frailty, cognitively normal

\section{INTRODUCTION}

Physical frailty is an age-related clinical syndrome that has been both cross-sectionally and prospectively associated with multiple adverse health outcomes, such as disability, hospitalization, and mortality, but in the initial stage, it is thought to be potentially reversible (i.e., pre-frail stage) $(1-4)$. Most research on frailty has been focused on the role of reduced physiological reserve in cardiovascular, skeletomuscular, and immune systems. However, there is also evidence that changes in the central nervous system may also contribute or evolve in parallel to frailty. 
Neuroimaging studies have shown that cerebrovascular damage, gray matter atrophy, as well as brain $\beta$-amyloid, may contribute to the pathophysiology of frailty. The severity of frailty status has been associated with a greater number of cerebral microbleeds (5), greater gray matter atrophy (6-8), higher white matter hyperintensity volume and infarcts (7-10), and higher brain $\beta$-amyloid burden $(11,12)$. A few longitudinal studies have shown that higher white matter hyperintensity volume or infarcts $(13,14)$, higher brain $\beta$-amyloid burden (11), and neuronal loss (14) are associated with the progression of frailty. A few studies examining regional brain atrophy with frailty suggest that brain areas important for motor function and execution may play a key role in frailty, such as selected areas in frontal, temporal, and parietal lobes, anterior cingulate cortex, subcortical areas, and cerebellum $(6,15)$.

Data on brain microstructure concerning frailty are sparse. Diffusion tensor imaging (DTI)-based brain microstructural measures may be sensitive to subtle age-related and vascular disease-related changes $(16,17)$ and can be used to monitor vascular disease progression (17). To date, only two DTI studies examined white matter microstructural characteristics with frailty $(18,19)$. These studies had relatively small samples and did not examine the microstructure of gray matter. Examining both gray and white matter microstructure across multiple regions of interest would allow us to determine the spatial distribution with frailty status, which may provide new insights into mechanisms and potential preventative strategies.

Examining sensitive neuroimaging markers, such as DTIbased microstructural integrity, and identifying specific brain areas connected with frailty or frailty risk in cognitively normal individuals may shed light on the spectrum of brain pathology underlying frailty. It may also identify new intermediate targets that can be tracked to verify the effectiveness of preventive interventions.

This exploratory study aimed to identify microstructural neuroimaging correlates of frailty, including both gray matter and white matter regions of interest (ROIs) in cognitively normal older adults. Our study poses an epidemiological question that is directly relevant for patient care: does DTI imaging provide important information to understand the contribution of the central nervous system to older patients' frailty status? We hypothesized that microstructural neuroimaging markers would be associated with frailty. We also hypothesized that microstructural correlates of frailty would be localized in brain areas important for motor function and execution, such as frontal, parietal and temporal lobes, anterior cingulate cortex, and subcortical areas.

\section{METHODS}

\section{Study Population}

We identified 670 cognitively normal participants aged 60 and older from the Baltimore Longitudinal Study of Aging (BLSA) and used the first concurrent data on frailty status and microstructural neuroimaging markers of interest by DTI. BLSA is a prospective cohort study with continuous enrollment that began in 1958 (20). Data from this study were collected between
June 2008 and December 2017. Exclusion criteria included diagnosis of mild cognitive impairment (MCI), Alzheimer's disease, and dementia. Diagnoses of cognitive impairment and dementia follow standard BLSA procedures, described previously (21). MCI was determined using the Peterson criteria (22). BLSA diagnose of dementia and Alzheimer's disease have continued to follow the Diagnostic and Statistical Manuel, third edition, revised (DSM-III-R) and the National Institute of Neurological and Communication Disorders and Stroke-Alzheimer's Disease and Related Disorders Association (NINCDS-ADRDA) criteria, respectively.

The BLSA protocol was approved by the Institutional Review Board of the National Institute of Environmental Health Sciences. Participants provided written informed consent at each BLSA visit.

\section{Frailty}

We use the Fried criteria (23) and a previously identified population-independent cutoff (24) to determine frailty status, including non-frail (scored as 0), pre-frail (scored 1-2), and frail (scored 3-5). In brief, the frail status was defined when three or more of the following criteria were present: unintentional weight loss ( $\geq 10 \mathrm{lbs}$ in the past year), self-reported exhaustion, slow gait speed, weakness (low grip strength), and low physical activity based on self-reported questionnaires (25). The previously validated population-independent cut-off approach has a good correlation and high agreement with the lowest-quintile approach $(r=0.84$, weighted kappa $=0.75)$ (24). Because the BLSA sample tends to be healthier than the general population, we used $1.0 \mathrm{~m} / \mathrm{s}$ to define slow gait speed (26). Components and cutoffs were presented in Supplementary Table 1.

\section{Imaging Acquisition}

Imaging data were acquired on $3 \mathrm{~T}$ Philips Achieva scanner (scanners 1 and 2 at the Kennedy Krieger Institute and scanner 3 at the National Institute on Aging) in Baltimore, Maryland. Imaging evaluations for each participant included a T1-weighted magnetization-prepared rapid gradient-recalled echo (MPRAGE) scan, an interleaved proton density and T2weighted dual-echo scan, a fluid-attenuated inversion recovery (FLAIR) scan, and two DTI scans. The MPRAGE protocol was as follows: number of slices $=170$, voxel size $=1 \mathrm{~mm} \times 1$ $\mathrm{mm} \times 1.2 \mathrm{~mm}$, reconstruction matrix $=256 \times 256$, flip angle $=8$ degrees and $\mathrm{TR} / \mathrm{TE}=6.5 \mathrm{~ms} / 3.1 \mathrm{~ms}$.

DTI acquisition protocol was identical for scanners 1 and 2: number of gradients $=32$, number of b0 images $=1$, $\max b$ factor $=700 \mathrm{~s} / \mathrm{mm}^{2}, \mathrm{TR} / \mathrm{TE}=6801 / 75 \mathrm{~ms}$, number of slices $=65$, voxel size $=0.83 \times 0.83 \times 2.2 \mathrm{~mm}$, reconstruction matrix $=256$ $\times 256$, acquisition matrix $=96 \times 95$, field of view $=212 \times$ $212 \mathrm{~mm}$, flip angle $=90^{\circ}$.

DTI acquisition protocol for scanner 3 was different from scanners 1 and 2: number of gradients $=32$, number of b0 images $=1$, $\max$ b-factor $=700 \mathrm{~s} / \mathrm{mm}^{2}$, TR $/ \mathrm{TE}=7454 / 75 \mathrm{~ms}$, number of slices $=70$, voxel size $=0.81 \times 0.81 \times 2.2 \mathrm{~mm}$, reconstruction matrix $=320 \times 320$, acquisition matrix $=116$ $\times 115$, field of view $=260 \times 260 \mathrm{~mm}$, flip angle $=90^{\circ}$. Each 
DTI acquisition included two b0 images, which were averaged in $\mathrm{k}$-space. The two separate DTI acquisitions with $\mathrm{NSA}=1$ were obtained and then combined offline (as explained in Image processing below) for an effective NSA $=2$ to improve signal-tonoise ratio (27).

\section{Diffusion Tensor Imaging Processing}

DTI processing follows the standard practice for tensor fitting and quality assessment and is explained in detail in earlier publications $(21,27)$. Briefly, the individual diffusion-weighted volumes were affine co-registered to a minimally weighted (b0) target to compensate for eddy current effects and physiological motion. The gradient tables were corrected for the identified rotational component using finite strain (28). To combine the two DTI sessions with different and unknown intensity normalization constants, each diffusion-weighted image was normalized by its own reference image before tensor fitting. QC was performed to remove scans with either excessive motion or images that had globally high diffusion measure bias after reviewing the distributions of QC summary statistics generated by our pipeline (27).

\section{Regions of Interest}

In this study, we focused on fractional anisotropy (FA) of white matter ROIs and mean diffusivity (MD) of gray matter ROIs.

To segment gray matter regions, we used multi-atlas registration with the BrainCOLOR protocol using 35 manually labeled atlases from NeuroMorphometrics (29). The labels of ROIs obtained from the T1 image for each visit were affine registered to the diffusion image and used to extract regionspecific average MD measures.
To segment white matter, the Eve White Matter atlas (30) was combined with corresponding WM labels from the multi-atlas segmentation (29), and an FA mapped MRI. The WM labels were then intersected with WM segmentation and the resulting labels are iteratively grown to fill the remaining WM space from the multi-atlas labels. The WM labels of ROIs obtained from the T1 image for each visit were affine registered to the FA image and used to extract region-specific average FA measures.

\section{Statistical Analysis}

Univariate correlations of participant characteristics with frailty status (non-frail, pre-frail, frail) were examined using Spearman correlation coefficients for continuous variables and chi-square tests for categorical variables as appropriate.

We identified DTI-based neuroimaging markers that were being univariately associated with frailty status (non-frail, prefrail, frail) using simple linear regression. We further adjusted for covariates that were important for brain health and/or frailty using multivariable linear regression. Covariates included age, sex, race, years of education, body mass index, and APOE e4 status.

For neuroimaging markers associated with frailty status at $p \leq 0.05$, we further examined differences between non-frail, pre-frail, frail groups using multivariable linear regression with "non-frail" being the reference group, adjusting for age, sex, race, education, body mass index, APOE e4 status, and also the scanner type. Dummy coded vectors were created to define "pre-frail" and "frail." In this exploratory analysis, significance was set at $p \leq 0.05$. Because vascular burden may contribute to both brain health and frailty status, we performed additional

TABLE 1 | Participant characteristics $(n=670)$.

\begin{tabular}{|c|c|c|c|c|}
\hline & $\begin{array}{l}\text { Non-frail } \\
(n=362)\end{array}$ & $\begin{array}{l}\text { Pre-frail } \\
(n=279)\end{array}$ & $\begin{array}{c}\text { Frail } \\
(n=29)\end{array}$ & \multirow[t]{2}{*}{$p$-value } \\
\hline & \multicolumn{3}{|c|}{ mean \pm SD or $N(\%)$} & \\
\hline \multicolumn{5}{|l|}{ Demographics } \\
\hline Age, years & $70.2 \pm 6.9$ & $77.2 \pm 7.8$ & $81.3 \pm 8.0$ & $<0.001$ \\
\hline Women & $185(51)$ & $168(60)$ & $21(72)$ & 0.01 \\
\hline Body mass index, kg/m² & $27.2 \pm 4.5$ & $26.8 \pm 4.4$ & $27.2 \pm 4.6$ & 0.35 \\
\hline Years of education & $17.7 \pm 2.6$ & $17.6 \pm 2.9$ & $17.0 \pm 2.9$ & 0.25 \\
\hline Cardiovascular disease & $27(7.5)$ & $26(9.3)$ & $4(13.8)$ & 0.41 \\
\hline \multicolumn{5}{|l|}{ Global neuroimaging markers } \\
\hline Total brain volume, $\mathrm{cm}^{3}$ & $1149 \pm 111$ & $1101 \pm 110$ & $1065 \pm 125$ & $<0.001$ \\
\hline Muscle strength, kg & $34.8 \pm 9.4$ & $26.0 \pm 8.6$ & $23.7 \pm 8.1$ & $<0.001$ \\
\hline High intensity exercise, kcal/week, median (Q1-Q3) & $\begin{array}{c}1,655 \\
(747-3045)\end{array}$ & $\begin{array}{c}1,076 \\
(111-2,297)\end{array}$ & $\begin{array}{c}69 \\
(29-314)\end{array}$ & $<0.001$ \\
\hline
\end{tabular}

Values of total white matter hyperintensities in $\mathrm{cm}^{3}$ and high intensity exercise in kcal per week were presented as median (Q1-Q3) due to their skewed distribution. P-values were based on Spearman correlation coefficients for continuous variables and chi-square tests for categorical variables. 
sensitivity analyses by further adjusting for the prevalence of cardiovascular disease. Cardiovascular disease was defined based on self-reported medical history, medication use, or clinical exams (31). Values of FA and MD were in standardized Z scores due to small values. Because studies reported women tend to have higher prevalence of frailty than men, we tested sex

TABLE 2 | Univariate and adjusted associations of frailty status (non-frail, pre-frail, frail) with mean diffusivity of gray matter regions of interest (ROIs).

\begin{tabular}{|c|c|c|c|}
\hline & $\begin{array}{l}\text { Gray matter } \\
\text { ROls }\end{array}$ & $\begin{array}{c}\text { Univariate } \\
\text { associations }\end{array}$ & $\begin{array}{c}\text { Adjusted } \\
\text { associations }\end{array}$ \\
\hline & & $\beta(95 \% \mathrm{Cl})$ & $\beta(95 \% \mathrm{Cl})$ \\
\hline \multirow[t]{6}{*}{ Frontal } & $\begin{array}{l}\text { Inferior frontal } \\
\text { gyrus }\end{array}$ & $0.273(0.144,0.403)$ & $-0.116(-0.244,0.012)$ \\
\hline & $\begin{array}{l}\text { Superior frontal } \\
\text { gyrus }\end{array}$ & $0.223(0.094,0.353)$ & $0.013(-0.128,0.153)$ \\
\hline & $\begin{array}{l}\text { Middle frontal } \\
\text { gyrus }\end{array}$ & $0.332(0.204,0.460)$ & $-0.018(-0.147,0.112)$ \\
\hline & $\begin{array}{l}\text { Medial frontal } \\
\text { cortex }\end{array}$ & $0.475(0.349,0.601)$ & $0.146(0.021,0.270)$ \\
\hline & $\begin{array}{l}\text { Supplementary } \\
\text { motor area }\end{array}$ & $0.361(0.234,0.489)$ & $0.024(-0.110,0.158)$ \\
\hline & Precentral gyrus & $0.254(0.125,0.384)$ & $-0.072(-0.208,0.064)$ \\
\hline \multirow[t]{4}{*}{ Parietal } & Postcentral gyrus & $0.265(0.135,0.394)$ & $-0.049(-0.184,0.086)$ \\
\hline & Precuneus & $0.451(0.324,0.577)$ & $0.015(-0.105,0.136)$ \\
\hline & Angular gyrus & $0.359(0.231,0.487)$ & $0.002(-0.118,0.122)$ \\
\hline & $\begin{array}{l}\text { Supramarginal } \\
\text { gyrus }\end{array}$ & $0.325(0.196,0.453)$ & $-0.048(-0.169,0.072)$ \\
\hline \multirow[t]{5}{*}{ Temporal } & Entorhinal cortex & $0.319(0.191,0.448)$ & $0.041(-0.091,0.173)$ \\
\hline & $\begin{array}{l}\text { Parahippocampal } \\
\text { gyrus }\end{array}$ & $0.465(0.339,0.591)$ & $0.074(-0.055,0.202)$ \\
\hline & $\begin{array}{l}\text { Inferior temporal } \\
\text { gyrus }\end{array}$ & $0.417(0.290,0.544)$ & $0.016(-0.098,0.129)$ \\
\hline & $\begin{array}{l}\text { Superior temporal } \\
\text { gyrus }\end{array}$ & $0.345(0.217,0.473)$ & $-0.056(-0.172,0.059)$ \\
\hline & $\begin{array}{l}\text { Middle temporal } \\
\text { gyrus }\end{array}$ & $0.445(0.319,0.572)$ & $-0.006(-0.109,0.098)$ \\
\hline \multirow[t]{3}{*}{ Occipital } & $\begin{array}{l}\text { Inferior occipital } \\
\text { gyrus }\end{array}$ & $0.353(0.225,0.481)$ & $-0.014(-0.135,0.107)$ \\
\hline & $\begin{array}{l}\text { Superior occipital } \\
\text { gyrus }\end{array}$ & $0.283(0.154,0.413)$ & $-0.111(-0.236,0.014)$ \\
\hline & $\begin{array}{l}\text { Middle occipital } \\
\text { gyrus }\end{array}$ & $0.319(0.190,0.447)$ & $0.024(-0.102,0.149)$ \\
\hline \multirow[t]{4}{*}{ Subcortical } & Hippocampus & $0.536(0.412,0.661)$ & $0.079(-0.030,0.188)$ \\
\hline & Putamen & $0.599(0.476,0.722)$ & $0.209(0.083,0.335)$ \\
\hline & Caudate & $0.489(0.363,0.614)$ & $0.157(0.022,0.292)$ \\
\hline & Thalamus & $0.498(0.372,0.623)$ & $0.127(-0.003,0.258)$ \\
\hline \multirow[t]{3}{*}{ Limbic } & $\begin{array}{l}\text { Anterior cingulate } \\
\text { cortex }\end{array}$ & $0.512(0.387,0.637)$ & $0.124(0.004,0.244)$ \\
\hline & $\begin{array}{l}\text { Middle cingulate } \\
\text { cortex }\end{array}$ & $0.304(0.175,0.433)$ & $-0.109(-0.236,0.018)$ \\
\hline & $\begin{array}{l}\text { Posterior cingulate } \\
\text { cortex }\end{array}$ & $0.560(0.436,0.683)$ & $0.057(-0.053,0.167)$ \\
\hline
\end{tabular}

Adjusted associations controlled for age, sex, race, education, BMI, and APOE e4. Bold numbers indicate associations at $p \leq 0.05$. Mean diffusivity values were standardized Z scores. differences by adding interaction terms of sex and frailty status in the model.

Due to the relatively older age of the frail group, we performed additional sensitivity analyses by selecting agematched participants from the non-frail and pre-frail groups with a 1:2 ratio (non-frail: 2; pre-frail: 2 ; frail: 1 ). We repeated the analyses in this aged-matched sample to confirm the robustness of our findings obtained in the whole sample.

\section{RESULTS}

Participant characteristics are presented in Table 1. Based on the Fried frailty criteria, $54 \%$ of this study sample was categorized as "non-frail," 41\% was "pre-frail," and 5\% was "frail." Those who were "frail" appeared to be older and more likely to be women,

TABLE 3 | Univariate and adjusted associations of frailty status (non-frail, pre-frail, frail) with fractional anisotropy of white matter regions of interest (ROls).

\begin{tabular}{|c|c|c|}
\hline \multirow[t]{2}{*}{ White matter ROIs } & $\begin{array}{c}\text { Univariate } \\
\text { associations }\end{array}$ & $\begin{array}{c}\text { Adjusted } \\
\text { associations }\end{array}$ \\
\hline & $\beta(95 \% \mathrm{Cl})$ & $\beta(95 \% \mathrm{Cl})$ \\
\hline $\begin{array}{l}\text { Genu of the corpus } \\
\text { callosum }\end{array}$ & $-0.511(-0.643,-0.380)$ & $-0.098(-0.232,0.036)$ \\
\hline $\begin{array}{l}\text { Body of the corpus } \\
\text { callosum }\end{array}$ & $-0.496(-0.628,-0.363)$ & $-0.164(-0.305,-0.023)$ \\
\hline $\begin{array}{l}\text { Splenium of the corpus } \\
\text { callosum }\end{array}$ & $-0.421(-0.554,-0.287)$ & $-0.114(-0.258,0.029)$ \\
\hline Uncinate fasciculus & $-0.161(-0.301,-0.022)$ & $-0.055(-0.210,0.100)$ \\
\hline $\begin{array}{l}\text { Superior longitudinal } \\
\text { fasciculus }\end{array}$ & $-0.135(-0.270,0.0002)$ & $0.024(-0.128,0.176)$ \\
\hline $\begin{array}{l}\text { Inferior fronto-occipital } \\
\text { fasciculus }\end{array}$ & $-0.287(-0.422,-0.152)$ & $-0.071(-0.222,0.079)$ \\
\hline $\begin{array}{l}\text { Superior } \\
\text { fronto-occipital } \\
\text { fasciculus }\end{array}$ & $-0.355(-0.488,-0.222)$ & $-0.166(-0.313,-0.018)$ \\
\hline $\begin{array}{l}\text { Anterior limb of internal } \\
\text { capsule }\end{array}$ & $-0.341(-0.478,-0.205)$ & $-0.101(-0.249,0.047)$ \\
\hline $\begin{array}{l}\text { Posterior limb of } \\
\text { internal capsule }\end{array}$ & $-0.084(-0.221,0.054)$ & $-0.021(-0.171,0.129)$ \\
\hline External capsule & $-0.319(-0.453,-0.185)$ & $-0.001(-0.146,0.143)$ \\
\hline $\begin{array}{l}\text { Posterior thalamic } \\
\text { radiation }\end{array}$ & $-0.237(-0.372,-0.101)$ & $-0.121(-0.275,0.033)$ \\
\hline $\begin{array}{l}\text { Anterior of corona } \\
\text { radiate }\end{array}$ & $-0.496(-0.627,-0.366)$ & $-0.091(-0.224,0.043)$ \\
\hline $\begin{array}{l}\text { Superior of corona } \\
\text { radiate }\end{array}$ & $-0.219(-0.355,-0.083)$ & $-0.064(-0.217,0.089)$ \\
\hline $\begin{array}{l}\text { Posterior of corona } \\
\text { radiate }\end{array}$ & $-0.032(-0.168,0.104)$ & $0.005(-0.148,0.158)$ \\
\hline $\begin{array}{l}\text { Cingulum hippocampal } \\
\text { part }\end{array}$ & $-0.211(-0.346,-0.075)$ & $0.033(-0.113,0.179)$ \\
\hline Cingulum cingulate part & $-0.270(-0.404,-0.136)$ & $-0.008(-0.150,0.135)$ \\
\hline
\end{tabular}

Adjusted associations controlled for age, sex, race, education, BMI, and APOE e4. Bold numbers indicate associations at $p \leq 0.05$. Fractional anisotropy values were standardized Z scores. 
and have lower education, lower total brain volume, and higher white matter hyperintensity volume (Table $\mathbf{1}$ ).

After adjustment for age, sex, race, education, body mass index, and APOE e4 status, a higher frail score was associated with higher MD of the medial frontal cortex, putamen, caudate, thalamus, and anterior cingulate cortex (Table 2). A higher frail score was also associated with lower FA of the body of the corpus callosum, and superior fronto-occipital fasciculus (Table 3).

Compared to the non-frail group, the frail group had higher MD of the medial frontal cortex, putamen, caudate, thalamus, and anterior cingulate cortex; they also had lower FA of the body of the corpus callosum and superior fronto-occipital fasciculus at a marginal significance $(p=0.051)$ after full adjustment (Table 4). There were no differences in other ROIs between frail and non-frail groups (Table 4).

Compared to the non-frail group, the pre-frail group had significantly higher MD of the putamen, lower FA of the superior fronto-occipital fasciculus, and a trend toward lower FA of the body of the corpus callosum $(p=0.075)$ after full adjustment (Table 4). There were no differences of MD in other gray matter ROIs or FA in other white matter ROIs between pre-frail and non-frail groups (Table 4).

Additional adjustment for cardiovascular disease did not substantially alter these associations (Supplementary Table 2). Interaction terms of sex and frailty status were not statistically significant (data not shown).

In an age-matched sensitivity analysis adjusted for the same set of covariates except for age, results remained substantially similar except the association with FA of the superior fronto-occipital fasciculus. The magnitude of standardized regression coefficients was higher compared to the original analysis, although some of the comparisons showed a marginal significance given a smaller sample size. Specifically, the differences between non-frail and frail remained significant in $\mathrm{MD}$ of the medial frontal cortex, putamen, thalamus, and anterior cingulate cortex. Differences in $\mathrm{MD}$ of caudate and FA of the body of the corpus callosum showed a trend toward significance ( $p=0.073$ and 0.061 , respectively) (Supplementary Table 3).

\section{DISCUSSION}

In this sample of cognitively normal older individuals, we demonstrate for the first time that both gray and white matter microstructure integrity is associated with frailty. Noteworthy, these neural correlates are localized in selected frontal and subcortical motor areas as well as the body of the corpus callosum. These results are not affected by adjustment for demographics and APOE e4 status. In particular, analyses conducted in an age-matched subsample confirmed the robustness of our findings.

Our DTI findings provide insight into mechanisms by which emerging brain microstructure damage, likely due to altered vascular integrity, may contribute to age-related frailty. Our findings may also explain frailty being a major risk factor of incident vascular dementia $(32,33)$. We advanced prior knowledge by including both gray and white matter microstructure, by determining the spatial distribution of microstructure with frailty, and by focusing on cognitively normal older adults.

Of white matter ROIs examined, we found that fractional anisotropy of the corpus callosum was associated with frailty, which was in line with one previous report (18). We further identified the body of corpus callosum being strongly associated with frailty status. We also observed a trend in superior frontooccipital fasciculus, which is in line with previous findings (19). Notably, two previous DTI studies using tract-based spatial statistics report other DTI parameters, such as axial diffusivity (AD) and mean diffusivity (MD) of the anterior limb of internal capsule and superior corona radiata, differ by frailty status. Using the ROI approach, we also observed that $\mathrm{AD}$ and $\mathrm{MD}$ of these two ROIs were associated with the total frail score (data not shown).

We are the first to demonstrate microstructural characteristics of gray matter underlying frailty. Our findings concerning the spatial distribution of gray matter microstructure with frailty are in line with previous brain volumetric and $\beta$-amyloid findings ( 6 , $12,15,34)$. Specifically, the medial frontal cortex is an identified area important for motor function and associated with lower extremity performance, perhaps due to its key role in executive

TABLE 4 | Differences of neuroimaging markers identified in Tables 2, 3 between frailty status groups.

\begin{tabular}{|c|c|c|c|c|}
\hline & Regions of interest & $\begin{array}{l}\text { Non-frail } \\
(n=362)\end{array}$ & $\begin{array}{l}\text { Pre-frail } \\
(n=279)\end{array}$ & $\begin{array}{c}\text { Frail } \\
(n=29)\end{array}$ \\
\hline & & Reference & $\beta(95 \% \mathrm{Cl})$ & $\beta(95 \% \mathrm{Cl})$ \\
\hline \multirow{3}{*}{$\begin{array}{l}\text { Gray matter mean diffusivity } \\
\text { (higher=worse) }\end{array}$} & Medial frontal cortex & - & $0.083(-0.063,0.228)$ & $0.482(0.149,0.815)$ \\
\hline & Putamen & - & $0.152(0.005,0.298)$ & $0.595(0.258,0.932)$ \\
\hline & Caudate & - & $0.086(-0.071,0.243)$ & $0.494(0.134,0.855)$ \\
\hline \multirow{2}{*}{$\begin{array}{l}\text { White matter fractional anisotropy } \\
\text { (lower = worse) }\end{array}$} & Body of corpus callosum & - & $-0.147(-0.308,0.015)$ & $-0.398(-0.798,0.002)$ \\
\hline & Superior fronto-occipital fasciculus & - & $-0.168(-0.336,-0.001)$ & $-0.400(-0.803,0.002)$ \\
\hline
\end{tabular}

Models were adjusted for age, sex, race, years of education, body mass index, scanner type, and APOE e4 status. Bold number reflects associations at $p \leq 0.05$. Mean diffusivity and fractional anisotropy values were standardized $Z$ scores. 
function. We also observed a strong association of MD in the anterior cingulate cortex. The anterior cingulate includes the cingulate motor areas and is involved in locomotion. Its structure and function are associated with gait performance (35-37). The strong associations of MD in subcortical motor areas, including putamen, caudate, thalamus, highlight the disrupted subcortical integrity may precede frailty risk and the development of frailty. Notably, the thalamus has an important role in motor control and coordination and also has a strong structural and functional connection to the striatum of putamen and caudate.

We did not observe any association in the temporal lobe or specifically the hippocampus, which has been reported in previous brain volumetric studies by MRI and CT $(6,15,34)$. This inconsistency may be due to differences in characteristics of study participants, imaging analytical approaches (voxel-based morphometry vs. ROIs), or frailty assessment.

Although differences in these microstructural neuroimaging markers were mostly present between frail and non-frail, there were also differences between prefrail and non-frail groups, such as putamen and the body of the corpus callosum. As the pre-frail stage is thought to be potentially revisable, strategies to slow or delay microstructural degradation in these selected areas in the absence of cognitive impairment might be considered.

Among limitations, we acknowledge the cross-sectional design which cannot establish the temporal sequence between brain microstructure and frailty. The BLSA sample tends to be healthier than the general population. The small number of frail cases may introduce potential bias. Using the Fried frailty criteria may have underestimated "pre-frail" and "frail" status in this sample. We would expect a stronger signal if multiple aspects of frailty are captured, especially in non-clinical, communitydwelling older populations. The modified definition items in the frailty criteria, such as leisure hour activities, may limit the ability to compare with other studies. This study has several strengths. First, the DTI atlas allowed us to investigate the detailed microstructural properties in both gray and white matter ROIs. Second, this sample community-dwelling older adults are well-characterized with rigorous adjudication of cognitive impairment, including dementia, and APOE e4 genotype. This allowed us to focus on frailty in the absence of cognitive impairment and to account for APOE e4 status. Third, the age-matched sensitivity analyses confirmed the robustness of our findings.

In conclusion, microstructural integrity of selected frontal and subcortical motor regions as well as the body of the

\section{REFERENCES}

1. Dedeyne L, Deschodt M, Verschueren S, Tournoy J, Gielen E. Effects of multi-domain interventions in (pre)frail elderly on frailty, functional, and cognitive status: a systematic review. Clin Interv Aging. (2017) 12:873-96. doi: 10.2147/CIA.S130794

2. Haider S, Grabovac I, Dorner TE. Effects of physical activity interventions in frail and prefrail community-dwelling people on frailty status, muscle strength, physical performance and muscle mass-a narrative review. Wien Klin Wochenschr. (2019) 131:244-54. doi: 10.1007/s00508-019-1484-7 corpus callosum may be critical neural substrates underlying frailty among cognitively normal older adults. Future studies on whether early changes in brain microstructure precedes the development of frailty are needed.

\section{DATA AVAILABILITY STATEMENT}

The raw data supporting the conclusions of this article will be made available by the authors, without undue reservation.

\section{ETHICS STATEMENT}

The studies involving human participants were reviewed and approved by Institutional Review Board of the National Institute of Environmental Health Sciences. The patients/participants provided their written informed consent to participate in this study.

\section{AUTHOR CONTRIBUTIONS}

QT designed the study, participated in the quality control for the DTI data, performed statistical analyses, and drafted the manuscript. OW performed quality control for the DTI data, provided feedback and suggestions to the analyses, and critically evaluated the manuscript. BL developed DTI analytical approaches, provided feedback and suggestions to the analyses, and critically evaluated the manuscript. SR collected data, provided guidance on analyses, and critically evaluated the manuscript. LF designed the study, collected data, provided guidance on analyses, and critically evaluated the manuscript. All authors contributed to the article and approved the submitted version.

\section{FUNDING}

This research was supported by Intramural Research Program of the National Institute on Aging.

\section{SUPPLEMENTARY MATERIAL}

The Supplementary Material for this article can be found online at: https://www.frontiersin.org/articles/10.3389/fmed. 2020.546344/full\#supplementary-material
3. Puts MTE, Toubasi S, Andrew MK, Ashe MC, Ploeg J, Atkinson $\mathrm{E}$, et al. Interventions to prevent or reduce the level of frailty in community-dwelling older adults: a scoping review of the literature and international policies. Age Ageing. (2017) 46:383-92. doi: 10.1093/ageing/a fw247

4. Vermeiren S, Vella-Azzopardi R, Beckwee D, Habbig AK, Scafoglieri A, Jansen B, et al. Frailty and the prediction of negative health outcomes: a meta-analysis. J Am Med Dir Assoc. (2016) 17:1163 el161. doi: 10.1016/j.jamda.2016.0 9.010 
5. Chung CP, Chou KH, Chen WT, Liu LK, Lee WJ, Chen LK, et al. Cerebral microbleeds are associated with physical frailty: a community-based study. Neurobiol Aging. (2016) 44:143-50. doi: 10.1016/j.neurobiolaging.2016.04.025

6. Chen WT, Chou KH, Liu LK, Lee PL, Lee WJ, Chen LK, et al. Reduced cerebellar gray matter is a neural signature of physical frailty. Hum Brain Mapp. (2015) 36:3666-76. doi: 10.1002/hbm.22870

7. Del Brutto OH, Mera RM, Cagino K, Fanning KD, Milla-Martinez MF, Nieves JL, et al. Neuroimaging signatures of frailty: a population-based study in community-dwelling older adults (the atahualpa project). Geriatr Gerontol Int. (2017) 17:270-6. doi: 10.1111/ggi.12708

8. Kant IMJ, de Bresser J, van Montfort SJT, Aarts E, Verlaan JJ, Zacharias $\mathrm{N}$, et al. The association between brain volume, cortical brain infarcts, and physical frailty. Neurobiol Aging. (2018) 70:247-53. doi: 10.1016/j.neurobiolaging.2018.06.032

9. Kant IMJ, Mutsaerts H, van Montfort SJT, Jaarsma-Coes MG, Witkamp TD, Winterer G, et al. The association between frailty and mRI features of cerebral small vessel disease. Sci Rep. (2019) 9:11343. doi: 10.1038/s41598-019-47731-2

10. Siejka TP, Srikanth VK, Hubbard RE, Moran C, Beare R, Wood A, et al. Frailty and cerebral small vessel disease: a cross-Sectional analysis of the tasmanian study of cognition and gait (TASCOG). J Gerontol A Biol Sci Med Sci. (2018) 73:255-60. doi: 10.1093/gerona/glx145

11. Maltais M, De Souto Barreto P, Hooper C, Payoux P, Rolland Y, Vellas B, et al. Association between brain beta-Amyloid and frailty in older adults. J Gerontol A Biol Sci Med Sci. (2019) 74:1747-52. doi: 10.1093/gerona/glz009

12. Yoon DH, Lee JY, Shin SA, Kim YK, Song W. Physical frailty and amyloid-beta deposits in the brains of older adults with cognitive frailty. J Clin Med. (2018) 7:169-80. doi: 10.3390/jcm7070169

13. Maltais M, de Souto Barreto P, Moon SY, Rolland Y, Vellas B, Group MDS. Prospective association of white matter hyperintensity volume and frailty in older adults. Exp Gerontol. (2019) 118:51-4. doi: 10.1016/j.exger.2019.01.007

14. Buchman AS, Yu L, Wilson RS, Schneider JA, Bennett DA. Association of brain pathology with the progression of frailty in older adults. Neurology. (2013) 80:2055-61. doi: 10.1212/WNL.0b013e318294b462

15. Gallucci M, Piovesan C, Di Battista ME. Associations between the frailty index and brain atrophy: the treviso dementia (TREDEM) registry. J Alzheimers Dis. (2018) 62:1623-34. doi: 10.3233/JAD-170938

16. Lockhart SN, DeCarli C. Structural imaging measures of brain aging. Neuropsychol Rev. (2014) 24:271-89. doi: 10.1007/s11065-014-9268-3

17. Alves GS, Sudo FK, Alves CEO, Ericeira-Valente L, Moreira DM, Engelhardt E, et al. Diffusion tensor imaging studies in vascular disease: a review of the literature. Dement Neuropsychol. (2012) 6:158-63. doi: 10.1590/S1980-57642012DN06030008

18. Avila-Funes JA, Pelletier A, Meillon C, Catheline G, Periot O, Trevin OFI, et al. Vascular cerebral damage in frail older adults: the aMImage study. J Gerontol A Biol Sci Med Sci. (2017) 72:971-7. doi: 10.1093/gerona/ glw347

19. Maltais M, de Souto Barreto P, Perus L, Mangin JF, Grigis A, Chupin $M$, et al. Prospective associations between diffusion tensor imaging parameters and frailty in older adults. J. Am. Geriatr. Soc. (2020) 68:1050-5. doi: 10.1111 /jgs.16456

20. Ferrucci L. The baltimore longitudinal study of aging (BLSA): a 50-yearlong journey and plans for the future. J Gerontol A Biol Sci Med Sci. (2008) 63:1416-9. doi: 10.1093/gerona/63.12.1416

21. Tian Q, Ferrucci L, Resnick SM, Simonsick EM, Shardell MD, Landman BA, et al. The effect of age and microstructural white matter integrity on lap time variation and fast-paced walking speed. Brain Imaging Behav. (2016) 10:697-706. doi: 10.1007/s11682-015-9449-6

22. Petersen RC, Smith GE, Waring SC, Ivnik RJ, Kokmen E, Tangelos EG. Aging, memory, and mild cognitive impairment. Int Psychogeriatr. (1997) 9 Suppl 1:65-9. doi: 10.1017/S1041610297004717

23. Fried LP, Tangen CM, Walston J, Newman AB, Hirsch C, Gottdiener J, et al. Frailty in older adults: evidence for a phenotype. J Gerontol A Biol Sci Med Sci. (2001) 56:M146-56. doi: 10.1093/gerona/56.3.M146

24. Saum KU, Muller H, Stegmaier C, Hauer K, Raum E, Brenner H. Development and evaluation of a modification of the fried frailty criteria using population-independent cutpoints. J Am Geriatr Soc. (2012) 60:21105. doi: 10.1111/j.1532-5415.2012.04192.x

25. Brach JS, Simonsick EM, Kritchevsky S, Yaffe K, Newman AB, Health A, et al. The association between physical function and lifestyle activity and exercise in the health, aging and body composition study. J Am Geriatr Soc. (2004) 52:502-9. doi: 10.1111/j.1532-5415.2004.52154.x

26. Fielding RA, Vellas B, Evans WJ, Bhasin S, Morley JE, Newman $A B$, et al. Sarcopenia: an undiagnosed condition in older adults. Current consensus definition: prevalence, etiology, and consequences. International working group on sarcopenia. J Am Med Dir Assoc. (2011) 12:249-56. doi: 10.1016/j.jamda.2011.01.003

27. Lauzon CB, Asman AJ, Esparza ML, Burns SS, Fan Q, Gao Y, et al. Simultaneous analysis and quality assurance for diffusion tensor imaging. PLoS ONE. (2013) 8:e61737. doi: 10.1371/journal.pone.0061737

28. Alexander DC, Pierpaoli C, Basser PJ, Gee JC. Spatial transformations of diffusion tensor magnetic resonance images. IEEE Trans Med Imag. (2001) 20:1131-9. doi: 10.1109/42.963816

29. Klein A, Dal Canton T, Ghosh SS, Landman B, Lee J, Worth A. Open labels: online feedback for a public resource of manually labeled brain images. In: 16th Annual Meeting for the Organization of Human Brain Mapping. Barcelona (2010).

30. Lim IA, Faria AV, Li X, Hsu JT, Airan RD, Mori S, et al. Human brain atlas for automated region of interest selection in quantitative susceptibility mapping: application to determine iron content in deep gray matter structures. NeuroImage. (2013) 82:449-69. doi: 10.1016/j.neuroimage.2013.05.127

31. Fabbri E, An Y, Zoli M, Simonsick EM, Guralnik JM, Bandinelli S, et al. Aging and the burden of multimorbidity: associations with inflammatory and anabolic hormonal biomarkers. J Gerontol A Biol Sci Med Sci. (2015) 70:63-70. doi: 10.1093/gerona/glu127

32. Avila-Funes JA, Carcaillon L, Helmer C, Carriere I, Ritchie K, Rouaud O, et al. Is frailty a prodromal stage of vascular dementia? Results from the three-city study. J Am Geriatr Soc. (2012) 60:1708-12. doi: 10.1111/j.1532-5415.2012.04 142.x

33. Solfrizzi V, Scafato E, Frisardi V, Seripa D, Logroscino G, Maggi S, et al. Frailty syndrome and the risk of vascular dementia: the italian longitudinal study on aging. Alzheimers Dement. (2013) 9:113-22. doi: 10.1016/j.jalz.2011.0 9.223

34. Nishita Y, Nakamura A, Kato T, Otsuka R, Iwata K, Tange C, et al. Links between physical frailty and regional gray matter volumes in older adults: a Voxel-Based morphometry study. J Am Med Dir Assoc. (2019) 20:1587-92 e1587. doi: 10.1016/j.jamda.2019.09.001

35. de Laat KF, Reid AT, Grim DC, Evans AC, Kotter R, van Norden AG, et al. Cortical thickness is associated with gait disturbances in cerebral small vessel disease. Neuroimage. (2012) 59:1478-84. doi: 10.1016/j.neuroimage.2011.0 8.005

36. la Fougere C, Zwergal A, Rominger A, Forster S, Fesl G, Dieterich M, et al. Real versus imagined locomotion: a [18F]-FDG pET-fMRI comparison. Neuroimage. (2010) 50:1589-1598. doi: 10.1016/j.neuroimage.2009.1 2.060

37. Tian Q, Chastan N, Bair WN, Resnick SM, Ferrucci L, Studenski SA. The brain map of gait variability in aging, cognitive impairment and dementia-A systematic review. Neurosci Biobehav Rev. (2017) 74(Pt A):149-62. doi: 10.1016/j.neubiorev.2017.01.020

Conflict of Interest: The authors declare that the research was conducted in the absence of any commercial or financial relationships that could be construed as a potential conflict of interest.

Copyright (C) 2020 Tian, Williams, Landman, Resnick and Ferrucci. This is an openaccess article distributed under the terms of the Creative Commons Attribution License (CC BY). The use, distribution or reproduction in other forums is permitted, provided the original author(s) and the copyright owner(s) are credited and that the original publication in this journal is cited, in accordance with accepted academic practice. No use, distribution or reproduction is permitted which does not comply with these terms. 